\title{
PERTURBATION OF EMBEDDED EIGENVALUES ${ }^{1}$
}

\author{
BY JAMES S. HOWLAND \\ Communicated by Peter Lax, August 5, 1971
}

In [4] a Weinstein-Aronszajn multiplicity theory for embedded eigenvalues arising from a certain type of "resonance" was developed. The results announced here continue the work of [4], and generalize results of [2] and [3] to embedded eigenvalues of arbitrary finite multiplicity $m$, and to perturbations of infinite rank. In particular, we are able to discuss certain operators of quantum mechanics. A notable feature of the case $m>1$ is the appearance of Puiseux series for the resonances, in analogy to their appearance in the perturbation theory of isolated eigenvalues of nonselfadjoint operators [6, Chapters 2 and 7].

1. Puiseux series for resonances. Let $T$ be a selfadjoint operator on a separable Hilbert space $\mathscr{H}$, with resolvent $G(z)=(T-z)^{-1}$, and let $\lambda_{0}$ be a point eigenvalue of $T$ of finite multiplicity $m$. Denote by $P$ the orthogonal projection on $\operatorname{ker}\left(T-\lambda_{0}\right)$. Let $A$ and $B$ be bounded commuting selfadjoint operators on $\mathscr{H}$, and define

$$
H(\kappa)=T+\kappa A B
$$

For real $\kappa, H(\kappa)$ is selfadjoint and we define $R(z, \kappa)=(H(\kappa)-z)^{-1}$. Let $\Omega$ be a neighborhood $\lambda_{0}$ in the complex plane, and assume that the operator $Q(z)=A G(z) B$ is bounded and has meromorphic continuations $Q^{ \pm}(z)$ from $\Omega^{ \pm}=\{z \in \Omega: \operatorname{Im} z>0\}$ to $\Omega$. There is then a simple pole of $Q^{+}(z)$ at $\lambda_{0}$ with residue $A P B$. The functions $Q^{+}(z)$ and $Q^{-}(z)$ will not agree on $\Omega$ if the eigenvalue $\lambda_{0}$ is embedded in the continuous spectrum of $T$. The operator

$$
Q_{1}(z, \kappa)=A R(z, \kappa) B
$$

also has meromorphic continuations $Q_{1}^{ \pm}(z, \kappa)$ given by

$$
I-\kappa Q_{1}(z, \kappa)=[I+\kappa Q(z, \kappa)]^{-1} .
$$

It is the poles of $Q_{1}^{+}(z, \kappa)$ that we refer to as the resonances of this perturbation problem.

The following was proved in $[4, \S 5]$.

AMS 1969 subject classifications. Primary 4748, 8147; Secondary 3577.

Key words and phrases. Perturbation theory, embedded eigenvalues, resonance, Puiseux series, spectral concentration, Schroedinger operator, Auger effect, Fermi's Golden Rule.

${ }^{1}$ This research was supported in part by ARO Grant DA-ARO-D-31-124-G1005. 
THEOREM 1. There is an analytic function $\Delta(z, \kappa)$ on a polydisc $\{(z, \kappa)$ : $\left.\left|z-\lambda_{0}\right|<\delta_{1},|\kappa|<\delta_{2}\right\}$ such that

(a) for $|\kappa|<\delta_{2}, \Delta(z, \kappa)$ has exactly $m$ zeros $z_{1}(\kappa), \ldots, z_{m}(\kappa)$ (repeated according to multiplicity) in $\left|z-\lambda_{0}\right|<\delta_{1}$, which are precisely the poles of $Q_{1}^{+}(z, \kappa)$ in $\left|z-\lambda_{0}\right|<\delta_{2}$. For $\kappa=0, z_{j}(0)=\lambda_{0}(j=1, \ldots, m)$.

(b) If for some real $\kappa, z_{j}(\kappa)$ is real, then $z_{j}(\kappa)$ is an eigenvalue of $H(\kappa)$ of multiplicity equal to the multiplicity $m_{j}(\kappa)$ of $z_{j}(\kappa)$ as a zero of $\Delta(z, \kappa)$.

The next theorem discusses the dependence of $z_{j}(\kappa)$ on $\kappa$.

THEOREM 2. The resonances $z_{1}(\kappa), \ldots, z_{m}(\kappa)$ may be labeled so that each $z_{j}(\kappa)$ has a Puiseux series expansion in $\kappa$. If

$$
z_{j}(\kappa)=\lambda_{0}+\alpha_{1} \omega^{j} \kappa^{1 / p}+\alpha_{2} \omega^{2 j} \kappa^{2 / p}+\cdots \quad(j=1, \ldots, p)
$$

is a given Puiseux cycle of resonances, where $\omega$ is a primitive pth root of unity, then either the series has the form

$$
z_{j}(\kappa)=\lambda_{0}+\alpha_{p} \kappa+\cdots+\alpha_{2 n p} \kappa^{2 n}+\alpha_{2 n p+1} \omega^{j} \kappa^{(2 n+1) / p}+\cdots
$$

where $\lambda_{0}, \alpha_{p}, \ldots, \alpha_{(2 n-1) p}$ are real and $\operatorname{Im} \alpha_{2 n p}>0$; or $p=1$ and all the coefficients $\alpha_{n}$ are real.

Moreover, the multiplicity $m_{j}(\kappa)$ is independent of $\kappa$ for $\kappa \neq 0$ and sufficiently small, and is the same for each element $z_{j}(\kappa)$ of a given Puiseux cycle.

In particular, if $z_{j}(\kappa)$ belongs to a Puiseux cycle with $p \geqq 2$, then $z_{j}(\kappa)$ is not real for all sufficiently small $\kappa \neq 0$. Thus any actual embedded eigenvalues of $H(\kappa)$ are analytic.

COROLlary. For real $\kappa \neq 0$ sufficiently small, the multiplicity of point eigenvalues in the interval $\left(\lambda_{0}-\delta_{1}, \lambda_{0}+\delta_{1}\right)$ is independent of $\kappa$. If $z_{j}(\kappa)$ is real for all sufficiently small $\kappa$, then $z_{j}(\kappa)$ is analytic in $\kappa$.

An example can be given in which an eigenvalue of multiplicity $m=2$ gives rise to a nonanalytic Puiseux expansion in powers of $\kappa^{1 / 2}$. The perturbation $A B$ in this example has rank 4.

Let $\phi_{1}, \ldots, \phi_{m}$ be an orthonormal basis of $P \mathscr{H}$ in which the selfadjoint operator $P A B P$ on $P \mathscr{H}$ is diagonal.

THEOREM 3. If the eigenvalues $A_{1}, \ldots, A_{m}$ of the operator PABP on P $\mathscr{H}$ are all distinct, then $z_{j}(\kappa)(j=1, \ldots, m)$ are analytic in $\kappa$, and

$$
z_{j}(\kappa)=\lambda_{0}+\kappa \lambda_{j}+\kappa^{2}\left(Q_{c}^{+}\left(\lambda_{0}\right) A \phi_{j}, B \phi_{j}\right)+O\left(\kappa^{3}\right) \quad(j=1, \ldots, m),
$$

where

$$
Q_{c}^{+}(z)=Q^{+}(z)-\left(\lambda_{0}-z\right)^{-1} A P B
$$


is the analytic continuation of $A G(z) P_{c} B$ to a neighborhood of $\lambda_{0}$, and $P_{c}=I-P$.

For real $\kappa$, this implies that to a first approximation

$$
-\operatorname{Im} z_{j}(\kappa)=\pi \kappa^{2}\left[d\left(E(\lambda) P_{c} V \phi_{j}, V \phi_{j}\right) / d \lambda\right]_{\lambda=\lambda_{0}} \quad(j=1, \ldots, m)
$$

where $V=A B$ and $T=\int \lambda d E(\lambda)$. This result is known to physicists as Fermi's Golden Rule.

2. Spectral concentration. The proof of the following result on spectral concentration involves a grouping of the resonances into "clusters" in such a way that each cluster behaves asymptotically like a single simple pole of $Q_{1}^{+}(z, \kappa)$ (cf. the construction in [5]).

THEOREM 4. For $j=1, \ldots, m$ and $\kappa$ real, choose $\delta_{j}(\kappa)$ such that $\delta_{j}(\kappa)=o(1)$ and $\operatorname{Im} z_{j}(\kappa)=o\left(\delta_{j}(\kappa)\right)$ as $\kappa \rightarrow 0$. Let

$$
S(\kappa)=\bigcup_{j=1}^{m}\left\{t: \operatorname{Re} z_{j}(\kappa)-\delta_{j}(\kappa)<t<\operatorname{Re} z_{j}(\kappa)+\delta_{j}(\kappa)\right\} .
$$

If $H(\kappa)=\int \lambda d E_{\kappa}(\lambda)$, then

$$
P=\operatorname{st}-\lim \int_{\kappa \rightarrow 0} d E_{\kappa(\kappa)}(\lambda) .
$$

3. The Auger phenomenon. The results above may be applied to the Schroedinger operator

$$
H(\kappa)=-\Delta+V_{1}(x)+V_{2}(y)+\kappa V_{3}(x-y)
$$

acting on functions $u(x, y) \in L_{2}\left(R_{6}\right)$, where $x, y \in R_{3}$ and $\Delta$ is the 6-dimensional Laplacian. The functions $V_{i}(x)$ are assumed to be measurable on $R_{6}$ and to satisfy

$$
\left|V_{i}(x)\right| \leqq c e^{-\alpha|x|} \quad(i=1,2,3)
$$

One may describe $H(\kappa)$ as the Hamiltonian of a helium-like atom (with short range potentials), and the phenomenon discussed here is then analogous to the Auger effect.

The boundedness assumption on $A$ and $B$ can be weakened sufficiently to include potentials $V_{i}(x)$ which are locally $L_{2}$, and, in particular, the Yukawa potential.

Very recently, Simon [7] has announced an analyticity result, for simple multiplicity $(m=1)$, which applies to operators of the type $(*)$ where $V_{i}(x)$ are analytic functions of $|x|$, and includes, in particular, the Coulomb case. His work is based on results of Balslev and Combes [1]. 
ACKNOWLEDGMENT. This work was done at Stanford University while the author was on leave as an Associate in the Center for Advanced Studies at the University of Virginia. The author wishes to express his appreciation to the Center, and, for their hospitality, to Stanford and Professor R. S. Phillips.

\section{REFERENCES}

1. E. Balslev and J. Combes, Comm. Math. Phys. (to appear).

2. J. S. Howland, Perturbation of embedded eigenvalues by operators of finite rank, J. Math. Anal. Appl. 23 (1968), 575-584. MR 37 \# 5723.

3. - Embedded eigenvalues and virtual poles, Pacific J. Math. 29 (1969). 565-582. MR 40 \# 7862.

4. $\longrightarrow$ On the Weinstein-Aronszajn formula, Arch. Rational Mech. Anal. 39 (1970), $323-339$.

5. - Spectral concentration and virtual poles, Trans. Amer. Math. Soc. (to appear).

6. T. Kato, Perturbation theory for linear operators, Die Grundlehren der math. Wissenschaften, Band 132, Springer-Verlag, New York, 1966. MR 34 \# 3324.

7. B. Simon, Convergence of time-dependent perturbation theory for autoionizing states of atoms (to appear). 22903

Department of Mathematics, University of Virginia, Charlottesville, Virginia

Department of Mathematics, Stanford University, Stanford, California 94305 